\title{
Terminüberschreitung: Stellungnahme zur S1-Leitlinie
}

\author{
Deutsche Gesellschaft für Hebammenwissenschaft e.V.
}

\section{Die Deutsche Gesellschaft für Hebammenwissenschaft (DGHWi) e.V. setzt sich mit der überarbeiteten Fassung der S1-Leitlinie zum Vorgehen bei Terminüberschreitung und Übertragung auseinander. Begrifflichkeiten werden eingeordnet und Inhalte kritisch beurteilt sowie erklärend dargestellt.}

Mit freundlicher Genehmigung von Peters M. für die Deutsche Gesellschaft für Hebammenwissenschaften e.V. Stellungnahme zur überarbeiteten Fassung der S1-Leitlinie „Vorgehen bei Terminüberschreitung und Übertragung“ 02/ 2010 der Deutschen Gesellschaft für Gynäkologie und Geburtshilfe (DGGG) (19.10.2015). Z Hebammenwiss. 2015; 3 (2): 51-57.

Die Überarbeitung der S1 Leitlinie „Vorgehen bei Terminüberschreitung und Übertragung“ wird von der Deutschen Gesellschaft für Hebammenwissenschaft (DGHWi) aufgrund der Verunsicherungen der Praxis durch die Leitlinie in ihrer ersten Version (2010) ausdrücklich begrüßt. Die modifizierte Leitlinie stellt eine wichtige Orientierungshilfe für alle in der Praxis tätigen Ärzt/innen wie auch Hebammen dar. Wir freuen uns, dass einige unserer Kritikpunkte zur letzten Stellungnahme Einzug in die neue Version gefunden haben. Im Folgenden wird die Leitlinie durch die Sektion Schwangerenvorsorge kritisch gewürdigt.

\section{Methodik}

Die insgesamt 42 Seiten umfassende Leitlinie ist klar gegliedert und in ihrer Logik konsistent. Positiv hervorzuheben ist die sehr klare Beschreibung des Vorgehens bei Terminüberschreitung und Übertragung. Im Kapitel Problembeschreibung werden die Begriffe Termin- überschreitung und Übertragung klar definiert, leider ist die Trennschärfe der beiden Begriffe im Verlauf der Leitlinie nicht immer durchgängig gegeben. In der Beschreibung der Zielgruppe der Anwender/innen sind Hebammen, die unter anderem in der Schwangerschaftsvorsorge und der Geburtsbegleitung tätig sind, nicht erwähnt.

\section{Darstellung des Forschungsstandes}

In der Darstellung des Forschungsstandes werden Studien zu Häufigkeiten, möglichen Ursachen der Terminüberschreitung/Übertragung und zu möglichen Vorgehensweisen benannt. Wenngleich ein Methodenreport zur Verfügung gestellt wurde, bleibt unklar, welche Kriterien bei der Literaturauswahl zugrunde gelegt wurden. Eine systematische Literaturrecherche mit dokumentiertem und somit replizierbarem Vorgehen würde die Leitlinie bereichern. Beispielsweise fand in der vorliegenden Version der S1 Leitlinie „Vorgehen bei Terminüberschreitung und Übertragung“ der Review von Wennerholm et al. (2009) erneut keine Beachtung. Die Gründe hierfür lassen sich auf Grund der fehlenden Dokumentation nicht nachvollziehen.

An einigen Stellen der Leitlinie werden Sachverhalte als gut belegt dargestellt, ohne dass jedoch eine Quelle genannt wird. Dies ist beispielsweise bei der „Dauer einer normalen Schwangerschaft“ (S.4), bei der „Pathogenese der Auslösung des Geburtsvorganges“ (S.8), bei „verminderte Fruchtwassermenge und ihre Bedeutung für die Plazentainsuffizienz" (S.9) und bei der Genauigkeit der Terminbestimmung durch Ultraschall (S.31) der Fall. Darüber hinaus würde eine Trennung von Ergebnisdarstellung und Empfehlung die Lesbarkeit der Leitlinie verbessern (z.B. S. 15).

\section{Validität und Übertragbarkeit der Studienergebnisse}

Weder die Validität der Studienergebnisse noch die Übertragbarkeit der Studienergebnisse auf die aktuelle Versorgungsituation in Deutschland wird diskutiert. So ist beispielsweise eine Studie aus einem anderen zeitlichen Kontext nur bedingt auf die heutige Versorgungssituation übertragbar, vor allem, da sich die Genauigkeit der Terminbestimmung mittels Ultraschall, die intrauterine Diagnosestellung und der Gesundheitszustand der Schwangeren in den letzten Jahren entscheidend verändert haben. Diese Parameter sind für die Diagnose und die möglichen Folgen der Terminüberschreitung/Übertragung zentral.

Ebenso muss die Übertragbarkeit aus einem anderen kulturellen oder geografischen Kontext begründet werden. Es ist unklar, ob beispielsweise die amerikani- 
sche Schwangerenvorsorge und Geburtshilfe sowie die dort vorliegenden Risikofaktoren für einen IUFT mit der Situation in Deutschland gleichgesetzt werden können. Diese Diskussion findet nicht statt, obwohl aufbauend auf diesen Studien Empfehlungen für Deutschland ausgesprochen werden. Wünschenswert wäre dies bei den Studien von Yudkin (1987), Hilder (1998), Neilson (1998), Reddy (2006), Gülmezoglu (2012) und Martin et al. (2007) gewesen.

\section{Darstellung der Literatur und Zitation}

Ein präziserer Umgang mit der Darstellung der ausgewählten Literatur und der Interpretation von Grafiken wäre wünschenswert. So finden sich vereinzelt fehlende Übereinstimmungen zwischen Text und der dazugehörigen grafischen Darstellung. So wird im Text als Ergebnis der Studie von Reddy et al. (2006) „[...] eine deutliche Erhöhung des fetalen Risikos für einen IUFT zwischen $37+0$ und 41+6 SSW für alle Altersgruppen [...]“ thematisiert. Der dazugehörigen Grafik ist jedoch eine geringere Rate an IUFT in der 41+0 - 41+6 SSW im Vergleich zur 40+0 - 40+6 SSW zu entnehmen.

An mehreren Stellen der Leitlinie wird betont, dass mit zunehmendem Schwangerschaftsalter die Rate an operativen Geburten steigt. Die Grafik auf Seite 28 der Leitlinie zeigt jedoch, dass die Rate der Sectiones am 280. Schwangerschaftstag deutlich höher liegt als am 281., 282., 283., 284. und auch 285. Tag.

Auf einen sorgfältigen Umgang mit Zitationen sollte geachtet werden. Die Zitation der Arbeit von Weiss und Kollegen (2014) suggeriert eine Erhebung in ganz Deutschland. Der richtige Titel der Veröffentlichung lautet jedoch „Fetal mortality at and beyond term in singleton pregnancies in Baden-Wuerttemberg/ Germany 2004-2009“.

\section{Umgang mit Kausalität und Korrelation}

Ein wissenschaftlich korrekter Umgang mit den Konstrukten Korrelation und Kausalität wird erwartet. Es wurden in erster Linie Assoziationen, zum Teil auch physiologisch bzw. pathophysiologisch plausible Vorgänge gezeigt. Die Studiendesigns waren größtenteils retrospektiv und nicht adjustiert für Risikofaktoren. Insgesamt war kein Design dazu geeignet Kausalität nachzuweisen. Demnach ist nicht erwiesen, dass die Überschreitung des errechneten Termins ursächlich zu einer erhöhten kindlichen und mütterlichen Morbidität bzw. Mortalität führt.

Auffällig erscheint das Bestreben, die Rate an operativen Geburten und Sectiones in das Risikoprofil einer Terminüberschreitung/Übertragung mit aufzunehmen und somit einen kausalen Zusammenhang zwischen einer Terminüberschreitung/Übertragung und einer erhöhten Sectiorate her zu stellen. Da die Rate der Interventionen zu einem hohen Anteil vom Erfahrungswert des Behandelnden abhängt, dessen Vorgehen zudem auch äußeren Einflüssen unterliegt, ist das scheinbare Herleiten von kausalen Zusammenhängen bzw. das Suggerieren selbiger sehr fragwürdig. Die Berücksichtigung des „physician factor“ (DeMott and Sandmire 1990, 1992; Sandmire and DeMott 1994, 1996), darf bei der Interpretation von Studienergebnissen durchaus erwartet werden. Auch Outcome-Parameter wie höhergradige Dammrisse, auffällige Nabelschnur pHWerte oder ein protrahierter Geburtsverlauf sind nicht zwingend ursächlich durch die Terminüberschreitung bedingt, sondern können gleichwohl das Ergebnis von Interventionen sein.

\section{Fehlende Adjustierung}

Aus der Darstellung des Forschungsstandes wird sehr gut ersichtlich, dass die Terminüberschreitung/Übertragung als multifaktoriell bedingtes Geschehen zu bewerten ist. In der Leitlinie werden da- bei Risikofaktoren benannt, die nicht nur in Zusammenhang mit einem höheren Risiko der Terminüberschreitung/Übertragung zu sehen sind, sondern die auch, wie aus anderen Veröffentlichungen bekannt, in einem von der Schwangerschaftswoche unabhängigen statistischen Zusammenhang mit Tot- und Fehlgeburten stehen (Gardosi et al. 2013, Flenady et al. 2011, Frøen et al. 2001, Reddy et al. 2006). Vor diesem Hintergrund verwundert es, dass die fehlende Adjustierung von Risikofaktoren wie mütterliches Alter, Zigarettenkonsum, niedriger Sozialstatus und Übergewicht nicht kritisch erwähnt und reflektiert werden.

\section{Absolute und relative Risiken}

Zum besseren Verständnis wäre eine Angabe von absoluten Risiken und nicht von relativen Risiken wünschenswert. Beispielsweise wird die höhere Rate Neugeborener über $4000 \mathrm{~g}$ nach $42+0$ SSW mit 20-25\% angegeben. Bei einer relativen Angabe bleibt die Anzahl der betroffenen Individuen unklar (Gigerenzer et. al. 2007). Die Erkennung einer Makrosomie (S.31) wird mit einer Sensitivität von $80 \%$ angegeben. Wünschenswert wäre bei der Angabe einer Sensitivität auch die Angabe der Spezifität bzw. der Falsch-Positiv-Rate. Darüber hinaus fehlen auch hier Quellenangaben. Bei Pilalis et al. (2012) beträgt die Erkennungsrate von „large for gestational age“ Feten bei einer Spezifität von $75 \%$ immerhin $72,5 \%$, während sie bei einer Spezifität von $95 \%$ nur $34,3 \%$ ausmacht (LGA hier definiert als Gewicht über der 95ten Perzentile).

\section{Umgang mit statistischen Größen}

Bei den „Empfehlungen zur Diagnostik“ wird die Genauigkeit der Feststellung des Gestationsalters mittels Ultraschall anhand der Scheitel-Steiß-Länge im 1. Trimenon mit einem 90\% Vertrauensbereich auf \pm 3 Tage angegeben. Eine 
Quellenangabe, die die derzeitige Routineversorgungssituation widerspiegelt, wäre hierzu wünschenswert. Nach der Literaturübersicht der Leitlinie „Determination of Gestational Age by Ultrasound" der Society of Obstetricians and Gynaecologists of Canada scheint die Genauigkeit des Intervalls eher überschätzt zu werden (Butt und Lim 2014). Aktuelle Studien lassen bei einem 95\% Vertrauensbereich maximal auf ein \pm 5 Tage genaues Ergebnis bei Messung im ersten Trimenon schließen (Doubilet, 2013). Auf dieser Grundlage ist eine Empfehlung irritierend, die Abstufungen von jeweils 3 Tagen enthält $(41+0,41+3,41+6)$, wenngleich die Genauigkeit der Terminbestimmung mittels Ultraschall mit einem 90\% Vertrauensbereich maximal auf 3 Tage genau sein kann.

\section{Inhalt}

\section{Ausgewählte Literatur}

Trotz der ausführlich dargestellten Kritik der DGHWi (Stellungnahme zur Leitlinie von 2010) am Review von Gülmezoglu (2006), Reprint (2009), Reprint (2012) wurden die Empfehlungen zur Einleitung für den Zeitraum zwischen $41+0$ bis $41+6$ SSW erneut im Wesentlichen auf das Review von 2012 aufgebaut (S. 35). Auf den ersten Blick erscheint das Ergebnis der Metaanalyse eindeutig: In der Gruppe der Schwangeren mit Einleitungen verstarb lediglich ein Kind, in der Gruppe der Schwangeren mit abwartendem Vorgehen verstarben hingegen 14 Kinder.

Auf den zweiten Blick fallen jedoch die zahlreichen methodischen Schwächen der einbezogenen Studien sowie der zum Teil erheblich unterschiedliche geografische oder kulturelle sowie zeitliche Kontext auf. Vier der sechs Studien sind älter als zwanzig Jahre, sodass eine Übertragung auf den heutigen Kontext fraglich ist, beachtet man beispielsweise die Genauigkeit der Terminbestimmung, die Schwangerenvorsorge oder die Einleitungsmethoden. Ein kritischer Umgang mit der vorhandenen Literatur hätte diese Leitlinie sicher bereichert. Wünschenswert wäre eine Berücksichtigung der unterschiedlichen Definitionen einer Terminüberschreitung (in einer Spanne von 287 bis 294 Tagen), die als Basis der einzelnen Studien dienten (vergl. Wennerholm et al. 2009). Auch ist eine Totgeburt gemessen an der Stichprobengröße der einzelnen Studien ein zu seltenes Ereignis, um Schlüsse für eine adäquate Betreuung jenseits der 41+0 SSW zu ziehen (Wennerholm et al. 2009). Weitere Limitationen der Studien liegen im unterschiedlichen Versorgungskontext (Tab. 1).

Eine aktuelle Studie auf Grundlage der bundesdeutschen Perinataldaten (20042013) zeigt die niedrigste fetale Mortalität zwischen $37+0$ SSW und $39+6$ SSW mit 0,2 Totgeburten je 1000 fortbestehenden Schwangerschaften. „Zwischen der $41+0$ und $41+6$ SSW liegt die fetale Mortalität bei 0,6 und in der folgenden Woche dann bei 2,3. Ab der $42+6$ SSW ergibt sich eine Mortalitätsrate von 6,3/1000“ (Schwarz et al. 2015a). Damit steigt die fetale Mortalität zwischen der $41+0$ bis $41+6$ SSW im Vergleich zum Zeitraum zwischen der $37+0$ und der 39 + 6 SSW zwar an, ist jedoch bis zu $41+6$ SSW insgesamt sehr niedrig (Schwarz et al. 2015a).

Zwei aktuelle populationsbasierte Studien mit retrospektivem Design, eine aus Kanada und eine aus Deutschland, konnten keine Hinweise dafür erbringen, dass Unterschiede in den Einleitungsraten und Einleitungszeitpunkten die fetale Mortalität senken (Caughey 2015; Schwarz et al. 2015b). Es lässt sich festhalten, dass die derzeitige Evidenz keinen eindeutigen Unterschied in der fetalen und maternalen Mortalität zwischen den Betreuungsarten abwartendes Vorgehen und Einleitung zeigt. Auch ist nicht erwiesen, dass eine frühere Beendigung der Schwangerschaft durch Einleitung oder Sectio caesarea zu einem besseren Outcome unter den derzeitigen Versorgungsbedingungen führt. Somit gibt es keine verlässliche wissenschaftliche Grundlage zur Implementierung 
Tab. 1 Todesfälle und die Übertragung auf Deutschland limitierende Faktoren der Studien in der Metaanalyse Gülmezoglu (2012).

\begin{tabular}{|c|c|c|c|c|c|c|c|}
\hline $\begin{array}{l}\text { Erstautorln } \\
\text { der Studie }\end{array}$ & $\begin{array}{l}\text { Jahr der } \\
\text { Veröffent- } \\
\text { lichung }\end{array}$ & $\begin{array}{l}\mathrm{N}= \\
\text { Einleitung/ } \\
\text { Abwartend }\end{array}$ & $\begin{array}{l}\text { Perinatale } \\
\text { Todesfälle } \\
\text { inkl. Tot- } \\
\text { geburten } \\
\text { Einleitung/ } \\
\text { Abwartend }\end{array}$ & $\begin{array}{l}\text { Totgeburten } \\
\text { Einleitung/ } \\
\text { Abwartend }\end{array}$ & $\begin{array}{l}\text { Intervention und } \\
\text { Kontrollgruppe(n) } \\
\text { (limitiert die Über- } \\
\text { tragung auf die } \\
\text { Routineversorgung } \\
\text { in Deutschland) }\end{array}$ & $\begin{array}{l}\text { Versorgungs- } \\
\text { kontext } \\
\text { (limitiert } \\
\text { die Über- } \\
\text { tragung auf } \\
\text { die Routine- } \\
\text { versorgung in } \\
\text { Deutschland) }\end{array}$ & $\begin{array}{l}\text { Unter- } \\
\text { suchungs- } \\
\text { zeitraum } \\
\text { hinsichtlich } \\
\text { der SSW - } \\
\text { soweit an- } \\
\text { gegeben }\end{array}$ \\
\hline Bergsjö & 1989 & $94 / 94$ & $1 / 2$ & $0 / 0$ & $\begin{array}{l}\text { Einleitung (Eipol- } \\
\text { lösung, Amniotomie, } \\
\text { Oxytocin) gegenüber } \\
\text { Abwarten mit engen } \\
\text { Kontrollen }\end{array}$ & China & ab $42+0$ SSW \\
\hline Hannah & 1992 & $1701 / 1706$ & $0 / 2$ & $0 / 2$ & $\begin{array}{l}\text { Einleitung mit } \\
\text { intrazervikalem } \\
\text { Prostaglandin E2 } \\
\text { gegenüber Abwarten } \\
\text { mit Kickchart und } \\
\text { drei Mal CTG und FW } \\
\text { Messung }\end{array}$ & Kanada & $\begin{array}{l}41+0 \text { bis } \\
44+0 \text { SSW }\end{array}$ \\
\hline Heimstad & 2007 & $254 / 254$ & $0 / 1$ & $0 / 0$ & $\begin{array}{l}\text { Einleitung gegenüber } \\
\text { Untersuchung alle } \\
\text { drei Tage }\end{array}$ & Norwegen & ab $41+0$ SSW \\
\hline Henry & 1969 & $55 / 57$ & $0 / 2$ & $0 / 1$ & $\begin{array}{l}\text { Einleitung (Amnio- } \\
\text { tomie, Oxytocin) } \\
\text { gegenüber Amnio- } \\
\text { skopie }\end{array}$ & UK & ab $41+0$ SSW \\
\hline Herabutya & 1992 & $57 / 51$ & $0 / 1$ & $0 / 0$ & $\begin{array}{l}\text { Einleitung mit } \\
\text { Prostaglandin Gel } \\
\text { gegenüber Über- } \\
\text { wachung }\end{array}$ & Thailand & $\begin{array}{l}\text { Ab } 42+0 \\
\text { SSW }\end{array}$ \\
\hline Sahraoui & 2005 & $75 / 75$ & $0 / 1$ & $0 / 1$ & $\begin{array}{l}\text { Einleitung mit } \\
\text { Prostaglandin-Gel } \\
\text { gegenüber fragliche } \\
\text { Überwachung }\end{array}$ & Tunesien & $\begin{array}{l}41+0-41+6 \\
\text { SWS }\end{array}$ \\
\hline
\end{tabular}

oder Beibehaltung eines spezifischen Behandlungsmodells.

\section{Überwachung mittels CTG}

Der Wille, die diagnostischen und therapeutischen Interventionen bei Schwangeren ohne Risikoerhöhung zu vermeiden, wird sehr begrüßt. Unter dieser Vorgabe irritiert jedoch die Empfehlung, bei allen Schwangeren ab 40+0 Schwangerschaftswochen eine Überwachung mittels CTG zu befürworten, zumal dies der bestehenden Leitlinie zum CTG in der Schwangerschaft und während der Geburt widerspricht (Leitlinie CTG 2013). Die herangezogene wissenschaftliche Grundlage von Weiss (2014) erscheint angreifbar. Bei Weiss (2014) wird festgestellt, dass in Baden-Württemberg weniger IUFTs zu verzeichnen sind als in den USA. Dies wird u.a. darauf zurückgeführt, dass in Deutschland bereits ab $40+0$ SSW alle zwei Tage ein CTG durchgeführt wird. Dabei erscheinen die beiden Stichproben (DE, USA) hinsichtlich der kulturellen Unterschiede und der
Unterschiede im medizinischen Setting wenig vergleichbar. Zudem fehlt in der kalifornischen Vergleichsgruppe ein früher Ultraschall, der eine genauere Terminbestimmung zulässt. Ebenfalls fehlt eine Differenzierung der kalifornischen Vergleichsgruppe nach Bevölkerungsgruppe und Sozialstatus (ein Einflussfaktor des IUFTs). Das Argument „good clinical practice“, das sowohl den Aussagen der bestehenden Leitlinie (Leitlinie CTG 2013) als auch dem Grundsatz, unnötige Interventionen zu unterlassen, widerspricht, erscheint daher nicht ausreichend. 


\section{Informationen zur Einleitung und zu Methoden der Einleitung}

Generell sollten auch die Risiken einer Einleitung oder einer Sectio erwähnt werden. Durch Geburtseinleitung bedingte Probleme werden zum Teil unter 3.1 „Allgemeine Grundsätze“ (S.29) erwähnt. Sie sind jedoch nicht vollständig und nicht mit Quellenangaben versehen. So fehlen gänzlich Nebenwirkungen, wie zum Beispiel uterine Hyperstimulation, vermehrte Schmerzen, das Scheitern der Einleitung und eine uterine Ruptur bei vaginalem Prostaglandin E2 (NICE 2008); die Möglichkeit einer uterinen Hyperstimulation mit fetaler pathologischer Herzfrequenzveränderung, vermehrten Schmerzen und vermehrtem Mekoniumabgang bei oraler Verabreichung von Misoprostol (Alfirevic et al. 2014); oder eine Fruchtwasserembolie (Knight et al. 2010). Werden diese „,adverse effects“ jedoch nicht erwähnt, kann dies zu der Annahme führen, dass diese Optionen kein Risiko bergen. Ebenso fehlen Angaben zur „number needed to treat“.

Alternative Verfahren zur Einleitung wie Stimulation der Brust, Eipollösung, Rizinusöl, Nelkenöl oder Akupunktur (Kavanagh et al. 2001, 2005; Kelly et al. 2001) finden in der Leitlinie keine Erwähnung.

Die Wahrscheinlichkeit, dass nach Ablauf der $41+0$ SSW der Geburtsbeginn spontan innerhalb der nächsten drei Tage einsetzt, liegt bei $60 \%$, dass er innerhalb der folgenden sieben Tage einsetzt, bei $90 \%$. Bei Mehrgebärenden liegen die Prozentzahlen höher (Gardosi et al. 1997).

Auch diese Informationen wären wichtig für schwangere Frauen, die vor der Entscheidung Einleiten oder Abwarten stehen. Wünschenswert wäre eine Schilderung in einfacher und verständlicher Sprache, um die Beratung und Entscheidungsfindung zu unterstützen.

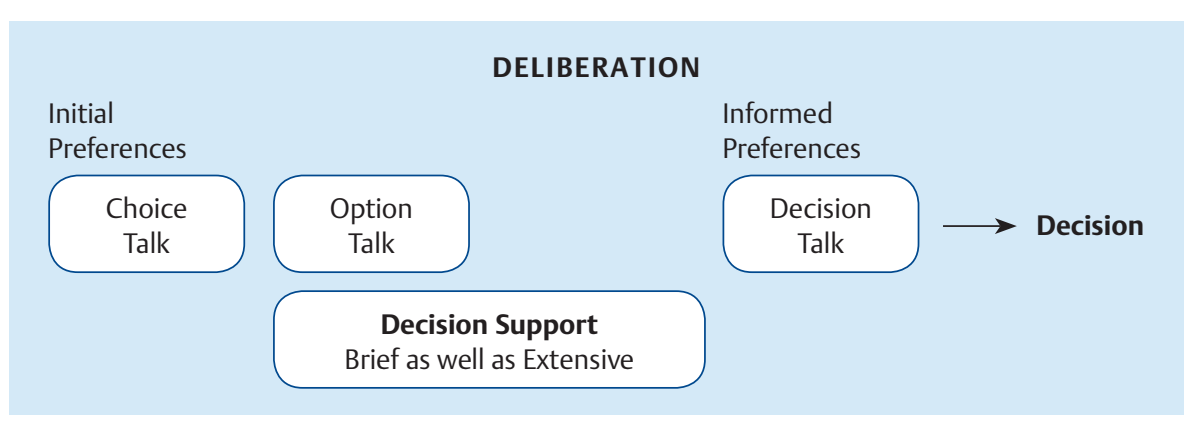

Abb. 1 Prozess des Shared Decision Making. (c) Aus Elwyn et al. 2012, S. 1365

\section{Nutzerinnenperspektive}

Wir begrüßen die „Allgemeinen Grundsätze“ (3.1) sehr. Auch wird gleich zu Beginn der Leitlinie gefordert, dass die Beratung der Schwangeren nach dem Konzept Informed Choice erfolgen muss. Die vorliegende Leitlinie spiegelt im Wesentlichen das Entscheidungsfindungsmodell des Informed Consent wider. Jedoch wird in den weiteren Ausführungen der Leitlinie diese Forderung nicht konsequent durchgehalten. Das Informed Choice Konzept impliziert unter anderem die Benennung der Durchführung sowie der Vor- und Nachteile aller Handlungsoptionen.

Der DGHWi ist das Recht der Schwangeren auf umfassende Information und ihre eigene Entscheidung über die Art und den Umfang der diagnostischen und therapeutischen Maßnahmen zentral. In unseren Augen ist die Situation, in der sich eine Schwangere bei der Überlegung zum medizinischen Umgang mit einer Terminüberschreitung/Übertragung befindet, prädisponiert für Informed Choice bzw. Shared Decision Making (SDM). Es besteht kein zeitlicher Handlungsdruck und es bestehen mehr als nur eine gerechtfertigte alternative Handlungsoption zum weiteren Umgang mit der Terminüberschreitung/Über-tragung (Elwyn et al. 2012). Elwyn et al. (2010) definieren Shared Decision Making als „an approach where clinicians and patients share the best available evidence when faced with the task of making decisions, and where patients are supported to consider options, to achieve informed preferences" (siehe Abb. 1).
Für eine nicht vorinformierte Schwangere ist es unmöglich zu entscheiden, welche Präferenzen sie hat und welche Entscheidung sie treffen möchte. SDM ist daher als ein Prozess anzusehen, bei dem die verschiedenen Optionen dargestellt und erläutert werden und die Schwangere schließlich bei ihrer Entscheidungsfindung unterstützt wird (Elwyn et al. 2012; Berger 2014). Aufbauend auf den gegebenen Informationen können sie über ihre Präferenzen beratschlagen und ihre Werte und Einstellungen reflektieren. Sie sollen so in das Gespräch mit einfließen, dass die Schwangere zu einer Entscheidung auf Grundlage sowohl der Evidenz als auch ihrer persönlichen Präferenzen gelangen kann (Elwyn et al. 2012). Entsprechend der international anerkannten Leitlinie „Induction of labour" des National Institute for Health and Clinical Excellence (NICE) darf bei dem Konzept des „Informed Choice“ die evidenzbasierte Darstellung der Vor- und Nachteile unterschiedlicher Vorgehensweisen bei Terminüberschreitung/ Übertragung erwartet werden. Die vorliegende Leitlinie konzentriert sich jedoch im Wesentlichen auf die Alternativen abwartende Haltung und Einleitung, ohne die unterschiedlichen Verfahren der Wehenstimulation näher kritisch zu beleuchten (Edwards et al. 2013).

Das Wesen der Beratung auf der Grundlagen des SDM ist eine Kommunikation, die die verschiedenen Möglichkeiten mit ihren evidenzbasierten Risiken und ihrem Nutzen darstellt, sodass sie von der Nutzerin verstanden werden kann (Berger 2014; Edwards et al. 2013; Elwyn et al. 2010). Dazu gehört auch, Unsicher- 
heiten in der Evidenz zu erklären (Berger 2014) und herauszufinden, was die Schwangere bereits weiß und ob dies im medizinischen Sinne korrekt ist (Elwyn et al. 2012).

\section{Sprachgebrauch}

Der Sprachgebrauch im Abschnitt 3 „Empfehlungen“ muss kritisch reflektiert werden. So wirken die Satzteile „(...) so kann unter Berücksichtigung der unter 3.1 bis 3.3 aufgeführten Empfehlungen bis 40+6 zugewartet werden“ und „Für den Fall, dass sich die Schwangere für ein Abwarten über den errechneten Termin hinaus entscheidet (...)“ verunsichernd. Der Satz „Unabhängig davon ist eine Schwangerschaftsbeendigung dann indiziert, wenn aktuelle Ergebnisse der Überwachung von Mutter und Kind eine Gefährdung anzeigen" (S.34) wurde unnötigerweise in fetter Schrift abgedruckt, da er ein selbstverständliches Vorgehens beschreibt. Diese Hervorhebung signalisiert eine Gefährdung, die nicht zwingend besteht.

Die Abstufungen „anbieten“, „empfehlen“ und ,indizieren“ sollten erklärt werden, da sie bei den Leser/innen zu Verwirrung führen können. Eine Versorgungsoption, die eine medizinische Fachkraft „anbietet“, sollte auch eine sein, die zu empfehlen ist. Ebenso wäre ein Satz begrüßenswert, der das Recht der Frau auf eine Informierte Entscheidungsfindung (Informed Choice) unterstreicht - entsprechend der NICE Guidelines (NICE 2008, Abschnitt: 1.2.1.3): „If a woman chooses not to have induction of labour, her decision should be respected. Healthcare professionals should discuss the woman's care with her from then on"

\section{Nichtwissen}

Auch fehlt die Thematisierung der Schwierigkeit, die tatsächliche Dauer einer menschlichen Schwangerschaft festzustellen. Laut einer Studie von Smith mit amerikanischen Schwangeren (2001), dauert eine Schwangerschaft im Mittel 283 Tage. Differenziert man nach der Parität, dauert sie im Mittel für Erstgebärende 283 und für Mehrgebärende im Mittel 284 Tage. Nach der Definition der Leitlinie kann eine Frau also bereits in die Kategorie Terminüberschreitung fallen, obwohl die mittlere Schwangerschaftsdauer nach Smith noch nicht erreicht ist. In diesem Zusammenhang sollte auch auf die Schwierigkeit hingewiesen werden, den genauen Zeitpunkt der Befruchtung und der Implantation und somit auch das Alter der Schwangerschaft zu bestimmen. Wilcox, Dunson und Baird (2000) dokumentierten in ihrer Stichprobe ein weit größeres Zeitfenster der weiblichen Fruchtbarkeit, das zwischen dem 6. und 21. Tag des Menstruationszyklus lag. Auch die Berechnung des Schwangerschaftsalters mittels Ultraschall ist in der Genauigkeit begrenzt (Vergleiche Abschnitt: Umgang mit statistischen Größen).

Wünschenswert wäre ein Kapitel, das ähnlich wie in den NICE-Guidelines akzentuiert darstellt, was wir nicht wissen. So ist beispielsweise die eigentliche Todesursache der Kinder in der Regel nicht bekannt. Deshalb ist auch unklar, ob eine Terminüberschreitung/Übertragung dafür verantwortlich gemacht werden kann. Ebenfalls sind die Auswirkungen des derzeitigen Vorgehens bei Terminüberschreitung mit Einleitungen auf die perinatale Mortalität, die Effektivität der Einleitungsdauer, die kurz- mittel- und langfristigen Nebenwirkungen der Einleitungsmethoden für Mutter und Kind, die Genauigkeit des Ultraschalls zur Terminbestimmung in der Routineversorgung, die Rolle der Diagnostik per CTG bei der Terminüberschreitung/Übertragung und einige weitere Themen unklar. Auch ist die Genauigkeit der Terminbestimmung im individuellen Fall oft unklar, sodass die darauf aufbauenden Empfehlungen im individuellen Fall fragwürdig sein können (Vergl. Butt und Lim 2014; Doubilet, 2013). Die Darstellung des Nicht-Wissens ist insbesondere wichtig, um den Anwender/innen zu vermitteln, dass die getroffenen Entscheidungen mit großer Unsicherheit getroffen wurden und um Wissenschaftler/ innen Ideen und Begründungen für Forschungsvorhaben zu liefern.

\section{Abschließende Empfehlung}

Wir empfehlen eine Überarbeitung der Leitlinie vor ihrem Ablaufdatum im Jahr 2017. Folgende Punkte sollten dabei aus unserer Sicht besonders berücksichtigt werden:

- Eine systematische Literaturrecherche mit transparenter Dokumenta- 
tion der Suchbegriffe und -zeiträume sowie die Angabe der recherchierten Datenbanken.

- Eine differenzierte Beurteilung der Literatur hinsichtlich ihrer Evidenzstärken (methodische Einschränkungen, systematische Verzerrungen, Generalisierbarkeit), auf welchen die Empfehlungen aufgebaut werden.

- Ein wissenschaftlicher Umgang mit statistischen Größen, welcher die Aussagekraft der einzelnen Messgrößen kritisch reflektiert, (nur) die zulässigen Schlüsse zieht und die Limitationen der Generalisierung offen legt.

- Die Verankerung des Konzepts Shared Decision Making in der Stellungnahme als Königsweg der Entscheidungsfindung für Schwangere und als Grundlage für eine Informierte Entscheidung.

- Eine differenzierte Darstellung der adverse effects und des Nichtwissens. Es muss deutlich zum Ausdruck gebracht werden, dass viele Empfehlungen in der Leitlinie unter (mehr oder weniger) Unsicherheit getroffen werden.

\section{Literatur:}

1 Alfirevic Z, Aflaifel N, Weeks A. Oral misoprostol for induction of labour. The Cochrane database of systematic reviews 2014; 6: DOI: 10.1002/14651858. CD001338.pub3

2 Berger Z. Navigating the unknown: shared decision-making in the face of uncertainty. Journal of general internal medicine 2015; 30 (5): 675-678

3 Bergsjo P, Huang GD, Yu SQ, Gao ZZ, Bakketeig LS. Comparison of induced versus non-induced labor in post-term pregnancy. A randomized prospective study. Acta obstetricia et gynecologica Scandinavica 1989; 68 (8): 683-687

4 Butt K, Lim K. Determination of gestational age by ultrasound. Journal of obstetrics and gynaecology Canada: JOGC = Journal d'obstetrique et gynecologie du Canada: JOGC 2014; 36 (2): 171-183
5 Caughey AB. Increasing rates of induction do not increase caesareans. BJOG: an international journal of obstetrics and gynaecology 2015; 122 (7): 981

6 DeMott RK, Sandmire HF. The Green Bay cesarean section study. I. The physician factor as a determinant of cesarean birth rates. American Journal of Obstetrics and Gynecology 1990; 162 (6): 1593-1599; discussion 1599-1602

7 DeMott RK, Sandmire HF. The Green Bay cesarean section study. II. The physician factor as a determinant of cesarean birth rates for failed labor. American Journal of Obstetrics and Gynecology 1992; 166 (6 Pt 1): 1799-1806; discussion 1806-1810

8 Doubilet PM. Should a first trimester dating scan be routine for all pregnancies? Seminars in perinatology 2013; 37 (5): 307-309

9 Edwards A, Unigwe S, Elwyn G, Hood K. Personalised risk communication for informed decision making about entering screening programs. The Cochrane database of systematic reviews 2003; (1): DOI: 10.1002/ 14651858.CD001865

10 Elwyn G, Frosch D, Thomson R, JosephWilliams N, Lloyd A, Kinnersley P et al. Shared decision making: a model for clinical practice. Journal of general internal medicine 2012; 27 (10): 1361-1367

11 Elwyn G, Laitner S, Coulter A, Walker E, Watson P, Thomson R. Implementing shared decision making in the NHS. BMJ (Clinical research ed.) 2010; 341: c5146

12 Flenady V, Koopmans L, Middleton P, Frøen JF, Smith GC, Gibbons K et al. Major risk factors for stillbirth in high-income countries: A systematic review and meta-analysis. The Lancet 2011; 377 (9774): 1331-1340

13 Frøen J, Arnestad M, Frey K, Vege Å, Saugstad OD, Stray-Pedersen B. Risk factors for sudden intrauterine unexplained death: Epidemiologic characteristics of singleton cases in Oslo, Norway, 1986-1995. American Journal of Obstetrics and Gynecology 2001; 184 (4): 694-702

14 Gardosi J, Madurasinghe V, Williams M, Malik A, Francis A. Maternal and fetal risk factors for stillbirth: population based study. BMJ (Clinical research ed.) 2013; 346: f108
15 Gardosi J, Vanner T, Francis A. Gestational age and induction of labour for prolonged pregnancy. BJOG: An international journal of O\&G 1997; 104 (7): 792-797

16 Gigerenzer G, Gaissmaier W, Kurz-Milcke E, Schwartz LM, Woloshin S. Helping doctors and patients to make sense of health statistics.: Psychological Science in the Public Interest.; 2007. Available from: URL:http://citrixweb.mpib-berlin. mpg.de/montez/upload/PaperLibrary/ GG_etAl_Helping_doctors-1.pdf

17 Gülmezoglu AM, Crowther CA, Middleton P, Heatley E. Induction of labour for improving birth outcomes for women at or beyond term. The Cochrane database of systematic reviews 2012; 6: DOI: 10.1002/14651858.CD004945.pub3

18 Hannah ME, Hannah WJ, Hellmann J, Hewson S, Milner R, Willan A. Induction of Labor as Compared with Serial Antenatal Monitoring in Post-Term Pregnancy: New England Journal of Medicine. N Engl J Med 1992; 326 (24): 1587-1592

19 Heimstad R, Romundstad PR, Hyett J, Mattsson L, Salvesen KA. Women's experiences and attitudes towards expectant management and induction of labor for post-term pregnancy. Acta obstetricia et gynecologica Scandinavica 2007; 8 6(8): 950-956

20 Henry GR. A controlled trial of surgical induction of labour and amnioscopy in the management of prolonged pregnancy. The Journal of obstetrics and gynaecology of the British Commonwealth 1969; 76 (9): 795-798

21 Herabutya Y, Prasertsawat PO, Tongyai T, Isarangura Na Ayudthya N. Prolonged pregnancy: the management dilemma. International journal of gynaecology and obstetrics: the official organ of the International Federation of Gynaecology and Obstetrics 1992; 37 (4): 253-258

22 Hilder L, Costeloe K, Thilaganathan B. Prolonged pregnancy: evaluating gestation-specific risks of fetal and infant mortality. BJOG: An International Journal of Obstetrics \& Gynaecology 1998; 105 (2): 169-173

23 Kavanagh J, Kelly AJ, Thomas J. Sexual intercourse for cervical ripening and induction of labour. The Cochrane database of systematic reviews 2001; (2): DOI: 10.1002/14651858.CD003093 
24 Kavanagh J, Kelly AJ, Thomas J. Breast stimulation for cervical ripening and induction of labour. The Cochrane database of systematic reviews 2005; (3): DOI: 10.1002/14651858.CD003392. pub2

25 Kelly AJ, Kavanagh J, Thomas J. Castor oil, bath and/or enema for cervical priming and induction of labour. The Cochrane database of systematic reviews 2001; (2): DOI: 10.1002/ 14651858.CD003099.pub2

26 Knight M, Tuffnell D, Brocklehurst P, Spark P, Kurinczuk JJ. Incidence and risk factors for amniotic-fluid embolism. Obstetrics and gynecology 2010; 115 (5): 910-917

27 Kreienbrock L, Pigeot I, Ahrens W. Epidemiologische Methoden. 5. Aufl. Heidelberg: Spektrum Akademischer Verlag; 2012. Available from: URL:http:// dx.doi.org/10.1007/978-3-8274-2334-4

28 Martin JA, Hamilton BE, Sutton PD, Ventura SJ, Menacker F, Kirmeyer S et al. Births: final data for 2005. National vital statistics reports : from the Centers for Disease Control and Prevention, National Center for Health Statistics, National Vital Statistics System 2007; 56 (6): 1-103

29 Neilson JP. Ultrasound for fetal assessment in early pregnancy. The Cochrane database of systematic reviews 2000; (2): DOI: 10.1002/14651858.CD000182

30 Induction of labour; 2008. Available from: URL:http://www.nice.org.uk/ guidance/cg70/chapter/guidance.

31 Olesen AW, Westergaard JG, Olsen J. Perinatal and maternal complications related to postterm delivery: a national register-based study, 1978-1993. American Journal of Obstetrics and Gynecology 2003; 189 (1): 222-227
32 Pilalis A, Souka AP, Papastefanou I, Michalitsi V, Panagopoulos P, Chrelias C et al. Third trimester ultrasound for the prediction of the large for gestational age fetus in low-risk population and evaluation of contingency strategies. Prenatal diagnosis 2012; 32 (9): 846-853

33 Reddy UM, Ko C, Willinger M. Maternal age and the risk of stillbirth throughout pregnancy in the United States. American Journal of Obstetrics and Gynecology 2006; 195 (3): 764-770

34 Sahraoui W, Hajji S, Bibi M, Nouira M, Essaidi H, Khairi H. Management of pregnancies beyond forty-one week's gestation with an unfavorable cervix. Journal de gynecologie, obstetrique et biologie de la reproduction 2005; 34 (5): 454-462

35 Sandmire HF, DeMott RK. The Green Bay cesarean section study. III. Falling cesarean birth rates without a formal curtailment program. American Journal of Obstetrics and Gynecology 1994; 170 (6): 1790-1799; discussion 17991802

36 Sandmire HF, DeMott RK. The Green Bay cesarean section study. IV. The physician factor as a determinant of cesarean birth rates for the large fetus. American Journal of Obstetrics and Gynecology 1996; 174 (5): 1557-1564

37 Schwarz C, Schäfers R, Loytved C, Heusser P, Abou-Dakn M, König T et al. Temporal trends in fetal mortality at and beyond term and induction of labor in Germany 2005-2012: data from German routine perinatal monitoring. Archives of gynecology and obstetrics 2015; DOI: 10.1007/s00404-015-3795-X

38 Schwarz C, Weiss E, Loytved C, Schäfers R, König T, Heusser P et al. Fetale Mortalität bei Einlingen ab Termin - eine Analyse bundesdeutscher Perinataldaten 2004-2013. Zeitschrift für Geburtshilfe und Neonatologie 2015; 219 (2): 81-85
39 Smith GC. Use of time to event analysis to estimate the normal duration of human pregnancy. Human Reproduction 2001; 16 (7): 1497-1500

40 Weiss E, Krombholz K, Eichner M. Fetal mortality at and beyond term in singleton pregnancies in Baden-Wuerttemberg/Germany 2004-2009. Archives of gynecology and obstetrics 2014; 289 (1): 79-84

41 Wennerholm U, Hagberg H, Brorsson B, Bergh C. Induction of labor versus expectant management for post-date pregnancy: is there sufficient evidence for a change in clinical practice? Acta obstetricia et gynecologica Scandinavica 2009; 88 (1): 6-17

42 Wilcox AJ, Dunson D, Baird DD. The timing of the "fertile window" in the menstrual cycle: day specific estimates from a prospective study. BMJ 2000; 32 (7271): 1259-1262

43 Yudkin PL, Wood L, Redman CW. Risk of unexplained stillbirth at different gestational ages. Lancet (London, England) 1987; 1 (8543): 1192-1194

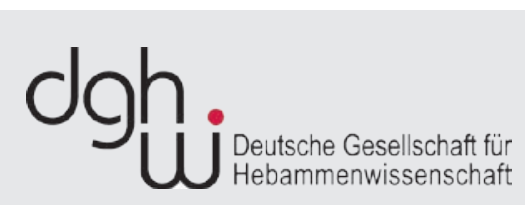

Deutsche Gesellschaft für Hebammenwissenschaften (DGHWi) http://www.dghwi.de/ geschaeftsstelle@dghwi.de 